\title{
Indirect and direct detection prospect for TeV dark matter in the MSSM-9
}

\author{
M. E. Cabrera Catalan ${ }^{* a}$ Shin'ichiro Ando, ${ }^{b}$ Christoph Weniger ${ }^{b}$ and Fabio \\ Zandanel $^{b}$ \\ ${ }^{a}$ Instituto de Física, Universidade de São Paulo \\ ${ }^{b}$ GRAPPA Institute, University of Amsterdam \\ E-mail: mcabrera@if.usp.br, s.ando@uva.nl, c.weniger@uva.nl, \\ F. Zandanel@uva.nl
}

\begin{abstract}
We perform a Bayesian analysis taking into account particle physics constraints, and upper limits on the scattering cross-section from direct detection experiments to investigate the prospects of indirect and direct dark matter searches for the minimal supersymmetric standard model with nine parameters (MSSM-9). We assume that the lightest neutralino provides all the dark matter through the thermal freeze-out mechanism. We find that the Cherenkov Telescope Array, CTA, together with XENON-1T will be able to probe a large fraction of the most probable regions of the MSSM-9: $\sim 1 \mathrm{TeV}$ higgsino-like and $\sim 3 \mathrm{TeV}$ wino-like neutralinos.
\end{abstract}

18th International Conference From the Planck Scale to the Electroweak Scale 25-29 May 2015

Ioannina, Greece

${ }^{*}$ Speaker. 


\section{Introduction}

The hunt for signatures of physics beyond the Standard Model of particle physics is going on at the LHC. Although there has been no claim of positive signatures, some outstanding conclusions can be drawn from several measurements, in particular for the Minimal Supersymmetric Standard Model (MSSM), one of this consequence comes from the mass measurement of the Higgs boson of about $126 \mathrm{GeV}[1,2]$. The rather high reported Higgs mass $m_{h}$ shifts the scale of supersymmetric masses to higher values with respect to what was expected based on naturalness arguments.

The Galactic center is one of the most promising places to search for signals from WIMP annihilation. Upper limits from modern gamma-ray instruments, such as the Large Area Telescope (LAT) aboard Fermi satellite, start to exclude this canonical annihilation cross-section for WIMP masses below $100 \mathrm{GeV}$ [3]. On the other hand the wino dark matter annihilation cross-section is significantly larger than the canonical value for WIMPs due to a non-perturbative effect known as Sommerfeld Enhancement (SE) [4, 5, 6, 7, 8], being already in some tension with searches for gamma-ray lines $[9,10,11]$.

In this proceeding we review the main result of the paper [12] where we studied the current status of the MSSM models with nine parameters (MSSM-9) and the prospects for CTA (indirect detection) and for XENON-1T (direct detection) experiments.

\section{Bayesian analysis of the MSSM-9}

There are several studies that have incorporated the all available experimental measurements to different parameterizations of the Minimal Supersymmetric Standard Model, e.g. Refs. [13, 14, $15,16,17,18,19,20]$. Various statistical approaches have been used to infer the most probable regions of these scenarios. Interestingly, when performing a proper Bayesian analysis, the finetuning penalization arises automatically from very basic statistical arguments, allowing to explore larger regions of the parameter space while taking the notion of naturalness automatically into account Ref. [21]. Electroweak fine-tuning arise as a Jacobian factor after including the Z-boson mass as an experimental value. In the same way fine-tuning associated to all the experimental measurements are included following this approach.

In order to evaluate the sensitivity on dark matter in a more generic context, we parameterize the MSSM with 10 fundamental parameters at the gauge coupling unification scale. After requiring the correct electroweak symmetry breaking, we end up with 9 effective parameters:

$$
\left\{s, M_{1}, M_{2}, M_{3}, m_{0}^{\tilde{q}}, m_{0}^{\tilde{l}}, m_{H}, A_{0}^{\tilde{q}}, A_{0}^{\tilde{l}}, \tan \beta, \operatorname{sgn}(\mu)\right\},
$$

where $s$ represents the SM nuisance parameters, $M_{1}, M_{2}, M_{3}$ are the gaugino masses, $m_{0}^{\tilde{q}}, m_{0}^{\tilde{l}}, m_{H}$ are the squark, slepton, and Higgs masses $\left(m_{H}=m_{H_{u}}=m_{H_{d}}\right)$, and $A_{0}^{\tilde{q}}$ and $A_{0}^{\tilde{l}}$ are the squark and slepton trilinear couplings. The sign of $\mu$ is fixed to +1 . All the soft parameters and $\mu$ defined at gauge coupling unification scale.

We take into consideration electroweak precision measurements [22], B-physics observables [23, 24, 25, 26, 27], the Higgs mass [1, 2], and constraints on the WIMP-nucleon scattering crosssection by XENON-100 [28] and LUX [29] , implementing the likelihood function as defined in ref. [30]. In particular, for Xenon100 we use the likelihood defined in [31]. The LUX limit is 

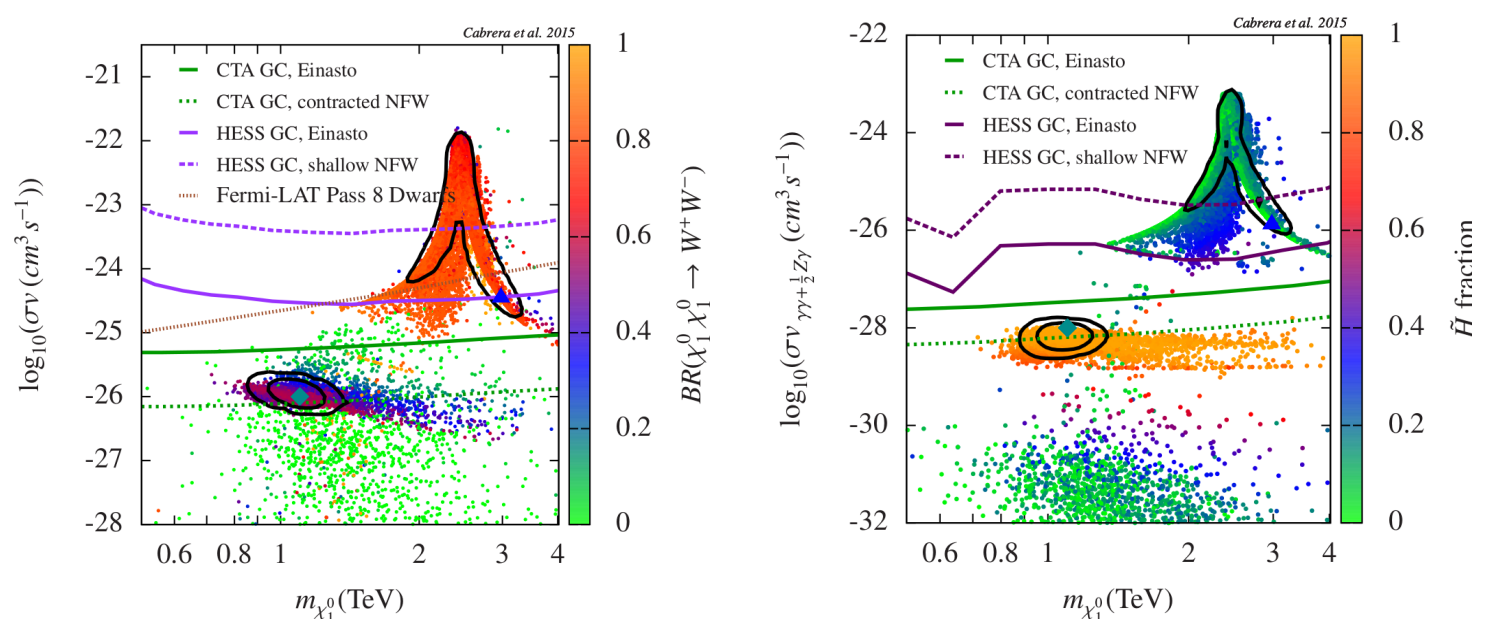

Figure 1: The contours represent $68 \%$ and $95 \%$ posterior probability credible regions in the plane neutralino mass versus total annihilation cross section (left) and annihilation into monochromatic photons (right). Colored points reproduce all the experimental observables within $2 \sigma$ of confidence level. The cyan diamond represent the pure higgsino case from [41, 6], and the blue triangle the pure wino case from $[9,33]$.

applied as a step function only for the $2 \sigma$ confidence level points. The Z-boson mass is effectively included adding a Jacobian factor.

In addition, we assume a scenario with a single dark matter component that is produced thermally in the early Universe, by including the measured relic density according to the Planck results [32]. For the relic density and $\langle\sigma v\rangle$ computation, we take the SE into account by creating a grid of the enhancement in the $M_{2}-\mu$ plane using the Hryczuk et al. computation method implemented in DarkSE $[8,33]$. For the computation of $\langle\sigma v\rangle$ in the present day we implemented a function in DarkSE to extract the enhancement for $v=10^{-3}$ from the Hryczuk et al. computation (we validated the results with the pure-wino case showed in [33]).

For the priors of the parameters, we adopted both standard and 'improved' log priors (S-log and I-log, respectively), defined in Refs. [13, 18]. In the following, we show results for the I-log priors only ${ }^{1}$.

For the numerical analysis, we use the SuperBayeS code [34], which uses the nested sampling algorithm implemented in Multinest [35], and integrates SoftSusy [36], SusyBSG [37], SuperIso [38], DarkSusy [39], MicrOMEGAs [40], and DarkSE [8] for the computation of the experimental observable.

\section{Results}

Left panel of Fig. 1 shows two-dimensional contours that represent $68 \%$ and $95 \%$ credible regions of the most relevant parameters for CTA: the dark matter neutralino mass and annihilation cross-section. The posterior has two peaks in the mass distribution. The largest peak locates

\footnotetext{
${ }^{1}$ The results obtained with S-log priors are very similar. Since the data, in particular the relic density constraint combined with the Higgs mass, turns out to be very constraining in the MSSM-9 when taking into account naturalness arguments into account.
} 


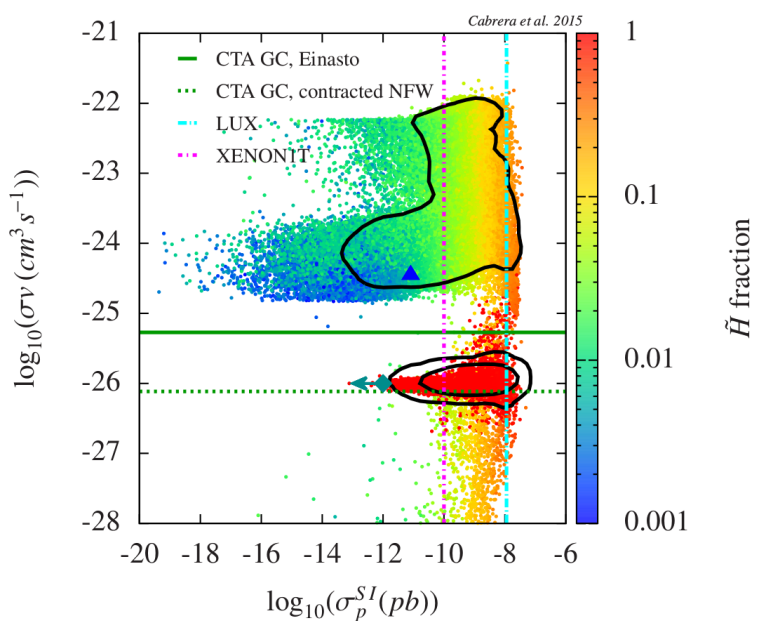

Figure 2: Similar to Fig. 1, but showing the total annihilation cross-section against the WIMP-proton spinindependent cross-section. The blue triangle and the cyan arrow are the theoretical values of the pure wino and pure higgsino case. The arrow indicates that the value of $\sigma_{p}^{S I}$ is a theoretical upper limit. Sensitivity lines from CTA and XENON-1T for $m_{\chi_{1}}=1 \mathrm{TeV}$.

around $1 \mathrm{TeV}$, where the neutralino mostly consists of higgsino. There is a weaker peak around 3 $\mathrm{TeV}$, where it is mostly wino.

Left panel of Figure 1 also shows, as points, regions in the parameter space that reproduce all experimental observables within $2 \sigma$ of confidence level. The $68 \%$ and $95 \%$ credibility regions show that it is much more likely to find neutralinos with a mass of $\sim 1 \mathrm{TeV}$ and $\sim 3 \mathrm{TeV}$, however, these contours not necessarily cover all the regions that respect the experimental observables. The scattered points outside the contours show regions that require more tuning to reproduce the experimental observables and, therefore, their integrated probability is small.

An additional region around hundreds $\mathrm{GeV}$, corresponding to bino-like neutralino, is not show in the figure. This region has small statistical weight and is not well explored in our scan, for this reason we show $m_{\chi_{1}^{0}}$ larger that $500 \mathrm{GeV}$ in our figures.

The colored points in left panel of Fig. 1 show the branching fraction of the lightest neutralino annihilating into $W^{+} W^{-}$. The colored points in right panel of Fig. 1 shows the higgsino fraction of the lightest neutralino. Note that points inside the $95 \%$ contour are dominantly higgsino-like and wino-like neutralinos.

\section{Prospects of Detection}

Figure 1 shows the predicted annihilation cross-section into continuum photons (dominated by $W^{+} W^{-}, Z Z$ and $\bar{q} q$ final states) and gamma-ray lines, respectively, compared to different experimental limits and reaches. Our fiducial density profile is given by an Einasto profile (with parameters $\alpha=0.17, \rho_{\odot}=0.4 \mathrm{GeV} / \mathrm{cm}^{3}$ and $r_{s}=20 \mathrm{kpc}$ ). The current upper limits on the Galactic center from HESS searches for gamma rays from $\bar{b} b$ final states (left panel of Fig. 1) [42] [43] and for gamma-ray lines (right panel of Fig. 1) [43] are already very tight. We find that the wino dark matter region around $3 \mathrm{TeV}$ is almost completely excluded by the HESS upper limits. 
However, that upper limits are still subject to uncertainties mainly related to the density profile [9]. To illustrate this point, we show in Fig. 1 how the HESS limits weaken when a shallower dark matter profile is adopted. To this end, we use a generalized NFW profile with an inner slope of $\gamma=0.7$ (and $r_{s}=20 \mathrm{kpc}, \rho_{\odot}=0.4 \mathrm{GeV} / \mathrm{cm}^{3}$ ), which is still in agreement with kinematic and microlensing observations [44]. In this case, the limits indeed are weakened and part of the wino best-fit region is still allowed. A similar effect will occur for cored profiles.

In left panel of Fig. 1, we also show the Fermi-LAT limits, corresponding to $\chi_{1}^{0} \chi_{1}^{0} \rightarrow b \bar{b}$ (limits for $W^{+} W^{-}$final states are very similar), from the observation of dwarf spheroidal galaxies from Ref. [45], which already include the uncertainties in the dark matter profile and can be hence considered robust (i.e., this represent the upper end of the uncertainty band). They exclude most of the wino parameter space.

The CTA sensitivities for both the total annihilation cross section derived for $\bar{b} b$ final states and $100 \mathrm{~h}$ observation time, assuming $1 \%$ systematics, [46] and for the gamma-ray lines [47] are shown in Fig. 1. These figures show that, for standard Einasto profiles, it will be challenging for CTA to reach the 1-TeV higgsino parameter space, unless background systematics are under control at the sub-percent level [46]. However, as explained above, baryonic effects could potentially increase the chances for a CTA discovery of higgsino dark matter as baryons can drag dark matter towards the Galactic center during their cooling, leading to a more cuspy profile [48]. To illustrate this effect, we additionally show in left panel of Fig. 1 the reach of CTA when a slightly contracted NFW profile, with an inner slope of $\gamma=1.3$ (and otherwise parameters as above), is adopted. In this case, CTA has the potential to rule out (or discover) a large part of the best-fit higgsino dark matter region.

Fig. 2 shows the most probable regions plotted for the annihilation cross-section and the spinindependent scattering cross-section $\sigma_{p}^{S I}$ at tree level. We also show higgsino neutralino (cyan diamond) and wino neutralino (blue triangle) one-loop computation performed by [49, 50]. In the pure higgsino case, the perturbative QCD and hadronic input $1 \sigma$-uncertainties allow only to set a maximum value for $\sigma_{p}^{S I}$, which is represented in the figure by an arrow. The colored points in Fig. 2 represent the higgsino composition of the lightest neutralino, and show how the value of $\sigma_{p}^{S I}$ decreases with the higgsino fraction in the wino-like region. In the region where $\sigma^{S I}$ is smaller that $\sim 10^{-11} \mathrm{pb}$ and $\sigma v$ larger than $10^{-25} \mathrm{~cm}^{3} \mathrm{~s}^{-1}$, the tree level $\sigma^{S I}$ does not give a realistic value, after including higher order corrections we would expect that those points will get $\sigma^{S I}$ values of $\sim 10^{-11} \mathrm{pb}$.

Figure 2 also shows the sensitivities of XENON-1T [51] and CTA [46] corresponding to $\chi_{1}^{0} \chi_{1}^{0} \rightarrow b \bar{b}$, both for $m_{\chi_{1}^{0}}=1 \mathrm{TeV}$, showing that both direct and indirect searches are very important for the potential discovery of $\mathrm{TeV}$ dark matter. Note also that the region around the almost-pure and pure wino and higgsino neutralinos will be probed by CTA only.

\section{References}

[1] ATLAS Collaboration Collaboration, G. Aad et. al., Observation of a new particle in the search for the Standard Model Higgs boson with the ATLAS detector at the LHC, Phys.Lett. B716 (2012) 1-29 [1207. 7214]. 
[2] CMS Collaboration Collaboration, S. Chatrchyan et. al., Observation of a new boson at a mass of 125 GeV with the CMS experiment at the LHC, Phys.Lett. B716 (2012) 30-61 [1207. 7235 ].

[3] Fermi-LAT Collaboration Collaboration, M. Ackermann et. al., Dark matter constraints from observations of 25 Milky Way satellite galaxies with the Fermi Large Area Telescope, Phys.Rev. D89 (2014) 042001 [1310.0828].

[4] J. Hisano, S. Matsumoto and M. M. Nojiri, Explosive dark matter annihilation, Phys.Rev.Lett. 92 (2004) 031303 [hep-ph/0307216].

[5] J. Hisano, S. Matsumoto, M. M. Nojiri and O. Saito, Non-perturbative effect on dark matter annihilation and gamma ray signature from galactic center, Phys.Rev. D71 (2005) 063528 [hep-ph/0412403].

[6] M. Cirelli, A. Strumia and M. Tamburini, Cosmology and Astrophysics of Minimal Dark Matter, Nucl.Phys. B787 (2007) 152-175 [0706.4071].

[7] N. Arkani-Hamed, D. P. Finkbeiner, T. R. Slatyer and N. Weiner, A Theory of Dark Matter, Phys.Rev. D79 (2009) 015014 [0810.0713].

[8] A. Hryczuk, R. Iengo and P. Ullio, Relic densities including Sommerfeld enhancements in the MSSM, JHEP 1103 (2011) 069 [1010.2172].

[9] T. Cohen, M. Lisanti, A. Pierce and T. R. Slatyer, Wino Dark Matter Under Siege, JCAP 1310 (2013) $061[1307.4082]$.

[10] J. Fan and M. Reece, In Wino Veritas? Indirect Searches Shed Light on Neutralino Dark Matter, JHEP 1310 (2013) 124 [1307. 4400].

[11] A. Hryczuk, I. Cholis, R. Iengo, M. Tavakoli and P. Ullio, Indirect Detection Analysis: Wino Dark Matter Case Study, JCAP 1407 (2014) 031 [1401.6212].

[12] M. E. Cabrera-Catalan, S. Ando, C. Weniger and F. Zandanel, Indirect and direct detection prospect for TeV dark matter in the nine parameter MSSM, Phys. Rev. D92 (2015), no. 3035018 [1503.00599].

[13] M. E. Cabrera, J. A. Casas and R. R. de Austri, The health of SUSY after the Higgs discovery and the XENON100 data, JHEP 1307 (2013) 182 [1212 . 4821].

[14] H. Silverwood, P. Scott, M. Danninger, C. Savage, J. Edsjãú et. al., Sensitivity of IceCube-DeepCore to neutralino dark matter in the MSSM-25, JCAP 1303 (2013) 027 [1210. 084 4].

[15] S. Akula, P. Nath and G. Peim, Implications of the Higgs Boson Discovery for mSUGRA, Phys.Lett. B717 (2012) 188-192 [1207. 1839].

[16] O. Buchmueller, R. Cavanaugh, M. Citron, A. De Roeck, M. Dolan et. al., The CMSSM and NUHM1 in Light of $7 \mathrm{TeV} \mathrm{LHC,} B_{s}$ to $\mu^{+} \mu^{-}$and XENON100 Data, Eur.Phys.J. C72 (2012) 2243 [1207. 7315].

[17] A. Fowlie, M. Kazana, K. Kowalska, S. Munir, L. Roszkowski et. al., The CMSSM Favoring New Territories: The Impact of New LHC Limits and a 125 GeV Higgs, Phys.Rev. D86 (2012) 075010 [1206.0264].

[18] M. E. Cabrera, A. Casas, R. R. de Austri and G. Bertone, LHC and dark matter phenomenology of the NUGHM, JHEP 1412 (2014) 114 [1311. 7152].

[19] O. Buchmueller, R. Cavanaugh, A. De Roeck, M. Dolan, J. Ellis et. al., The CMSSM and NUHM1 after LHC Run 1, Eur.Phys.J. C74 (2014), no. 62922 [1312 . 5250 ]. 
[20] L. Roszkowski, E. M. Sessolo and A. J. Williams, What next for the CMSSM and the NUHM: Improved prospects for superpartner and dark matter detection, JHEP 1408 (2014) 067 [1405.4289].

[21] M. Cabrera, J. Casas and R. Ruiz de Austri, Bayesian approach and Naturalness in MSSM analyses for the LHC, JHEP 0903 (2009) 075 [0812 . 0536].

[22] “The LEP Electroweak Working Group.” http://lepewwg.web.cern.ch/LEPEWWG.

[23] Heavy Flavor Averaging Group Collaboration, Y. Amhis et. al., Averages of B-Hadron, C-Hadron, and tau-lepton properties as of early 2012, 1207.1158.

[24] LHCb Collaboration Collaboration, R. Aaij et. al., Measurement of the $B_{s}^{0}-\bar{B}_{s}^{0}$ oscillation frequency $\Delta m_{s}$ in $B_{s}^{0} \rightarrow D_{s}^{-}(3) \pi$ decays, Phys.Lett. B709 (2012) 177-184 [1112.4311].

[25] BaBar Collaboration Collaboration, B. Aubert et. al. Phys.Rev.Lett. 100 (2008) 021801 [0709.1698].

[26] FlaviaNet Working Group on Kaon Decays Collaboration, M. Antonelli et. al., Precision tests of the Standard Model with leptonic and semileptonic kaon decays, 0801.1817.

[27] LHCb Collaboration Collaboration, R. Aaij et. al., First Evidence for the Decay $B_{s}^{0} \rightarrow \mu^{+} \mu^{-}$, Phys.Rev.Lett. 110 (2013), no. 2021801 [1211.2674].

[28] XENON100 Collaboration Collaboration, E. Aprile et. al., Dark Matter Results from 225 Live Days of XENON100 Data, Phys.Rev.Lett. 109 (2012) 181301 [1207. 5988].

[29] LUX Collaboration Collaboration, D. Akerib et. al., First results from the LUX dark matter experiment at the Sanford Underground Research Facility, Phys.Rev.Lett. 112 (2014) 091303 [1310.8214].

[30] R. R. de Austri, R. Trotta and L. Roszkowski, A Markov chain Monte Carlo analysis of the CMSSM, JHEP 0605 (2006) 002 [hep-ph / 0602028 ].

[31] G. Bertone, D. G. Cerdeno, M. Fornasa, R. Ruiz de Austri, C. Strege et. al., Global fits of the cMSSM including the first LHC and XENON100 data, JCAP 1201 (2012) 015 [1107. 1715].

[32] Planck Collaboration, P. Ade et. al., Planck 2013 results. XVI. Cosmological parameters, Astron.Astrophys. 571 (2014) A16 [1303.5076].

[33] A. Hryczuk and R. Iengo, The one-loop and Sommerfeld electroweak corrections to the Wino dark matter annihilation, JHEP 1201 (2012) 163 [1111.2916].

[34] C. Strege, G. Bertone, G. Besjes, S. Caron, R. Ruiz de Austri et. al., Profile likelihood maps of a 15-dimensional MSSM, JHEP 1409 (2014) 081 [1405. 0622].

[35] F. Feroz, M. Hobson and M. Bridges, MultiNest: an efficient and robust Bayesian inference tool for cosmology and particle physics, Mon.Not.Roy.Astron.Soc. 398 (2009) 1601-1614 [0 809.3437 ].

[36] B. Allanach, SOFTSUSY: a program for calculating supersymmetric spectra, Comput.Phys. Commun. 143 (2002) 305-331 [hep-ph/ 0104145$].$

[37] G. Degrassi, P. Gambino and P. Slavich, SusyBSG: A Fortran code for BR $[B \rightarrow X(s) \gamma]$ in the MSSM with Minimal Flavor Violation, Comput.Phys.Commun. 179 (2008) 759-771 [0 712 . 32 65].

[38] F. Mahmoudi, SuperIso v2.3: A Program for calculating flavor physics observables in Supersymmetry, Comput.Phys.Commun. 180 (2009) 1579-1613 [0808. 3144 ]. 
[39] P. Gondolo, J. Edsjo, P. Ullio, L. Bergstrom, M. Schelke et. al., DarkSUSY: Computing supersymmetric dark matter properties numerically, JCAP 0407 (2004) 008 [astro-ph/0406204].

[40] G. Belanger, F. Boudjema, A. Pukhov and A. Semenov, MicrOMEGAs: A Program for calculating the relic density in the MSSM, Comput.Phys.Commun. 149 (2002) 103-120 [hep-ph/0112278].

[41] S. Profumo, TeV gamma-rays and the largest masses and annihilation cross sections of neutralino dark matter, Phys.Rev. D72 (2005) 103521 [astro-ph/ 0508628 ].

[42] HESSCollaboration Collaboration, A. Abramowski et. al., Search for a Dark Matter annihilation signal from the Galactic Center halo with H.E.S.S, Phys.Rev.Lett. 106 (2011) 161301 [1103. 3266].

[43] H.E.S.S. Collaboration Collaboration, A. Abramowski et. al., Search for Photon-Linelike Signatures from Dark Matter Annihilations with H.E.S.S., Phys.Rev.Lett. 110 (2013) 041301 [1301.1173].

[44] F. Iocco, M. Pato, G. Bertone and P. Jetzer, Dark Matter distribution in the Milky Way: microlensing and dynamical constraints, JCAP 1111 (2011) 029 [1107.5810].

[45] Fermi-LAT Collaboration, M. Ackermann et. al., Searching for Dark Matter Annihilation from Milky Way Dwarf Spheroidal Galaxies with Six Years of Fermi-LAT Data, 1503.02641.

[46] H. Silverwood, C. Weniger, P. Scott and G. Bertone, A realistic assessment of the CTA sensitivity to dark matter annihilation, 1408.4131.

[47] L. Bergstrom, G. Bertone, J. Conrad, C. Farnier and C. Weniger, Investigating Gamma-Ray Lines from Dark Matter with Future Observatories, JCAP 1211 (2012) 025 [1207. 6773].

[48] F. Prada, A. Klypin, J. Flix, M. Martínez and E. Simonneau, Dark Matter Annihilation in the Milky Way Galaxy: Effects of Baryonic Compression, Physical Review Letters 93 (Dec., 2004) 241301 [astro-ph/0401512].

[49] R. J. Hill and M. P. Solon, WIMP-nucleon scattering with heavy WIMP effective theory, Phys.Rev.Lett. 112 (2014) 211602 [1309.4092].

[50] R. J. Hill and M. P. Solon, Standard Model anatomy of WIMP dark matter direct detection II: QCD analysis and hadronic matrix elements, Phys.Rev. D91 (2015), no. 4043505 [1409.8290].

[51] XENON1T collaboration Collaboration, E. Aprile, The XENON1T Dark Matter Search Experiment, Springer Proc.Phys. C12-02-22 (2013) 93-96 [120 6 . 6288]. 